suggest that the FDA alone controls access to experimental drugs, but major barriers to access lie beyond the agency's jurisdiction. Manufacturers, for instance, worry about liability, and physicians may not seek such drugs for fear of the FDA paperwork. In addition, Medicare, Medicaid, and private insurers generally will not pay for experimental drugs.

Pharmaceutical-industry representatives also express other reservations. "One of the biggest limitations is manufacturing capacity," says Scott Lassman of the Pharmaceutical Research and Manufacturers of America. "Especially in very early phases, the company may still be working out how to manufacture the product." Although the bill proposes allowing companies to charge for tier 1 drugs, says Lassman, they certainly couldn't charge full price. More important, "the whole purpose of large clinical trials is to fully evaluate benefits and risks," argues Frank Rockhold, an executive at GlaxoSmithKline, "and short-changing that is not in patients' best interests."

Nonetheless, the recent actions have apparently stimulated efforts to broaden access to experimental drugs within the current system, especially in light of increased demand from patients. "We've seen a significant increase in the treatment INDs that are requested," says Scott Gottlieb, the FDA's deputy commissioner for medical and scientific affairs. "The agency has generally been aggressive in granting those," usually acting within 24 to 48 hours on physicians' IND requests and encouraging companies to establish broader treatment IND programs if there is considerable demand for a drug. According to Gottlieb, the agency is working to clarify the process of requesting access to experimental drugs, in part by providing standardized application forms and an interface on the FDA's Web site.

This spring, the National Coalition for Cancer Survivorship and the American Society for Clinical Oncology petitioned the FDA to issue guidance to the pharmaceutical industry on standards for expanded-access programs; they suggested criteria for deciding when such programs are appropriate, ways to ensure equitability, and approaches to data collection and informed consent. Gottlieb says that the FDA has created a task force to respond to the petition and is clarifying its rules regarding what companies may charge. The FDA is permitted to approve compassionate use only for diseases in which evidence suggests that a given medication may have efficacy. However, the agency hopes to persuade companies to sponsor "simple, large, nonrandomized, open-access trials" for certain drugs that are in phase 3 trials, or possibly late in phase 2 trials, to make them available to more patients while providing additional data. "We think that you can have an approval process that is rigorous ... even though you have parallel mechanisms to allow broader access to a drug that has shown activity and promise," Gottlieb says.

An interview with Mr. William Schultz, a partner at the law firm Zuckerman Spaeder and a former deputy commissioner for policy at the FDA, can be heard at www.nejm.org.

Dr. Okie is a contributing editor of the Journal.

1. Society for Clinical Trials Board of Directors. The Society for Clinical Trials opposes US legislation to permit marketing of unproven medical therapies for seriously ill patients. Clin Trials 2006;3:154-7.

\title{
Measles in the United States, 2006
}

\author{
E. Kim Mulholland, M.D.
}

Related article, page 447

The control and virtual elimination of measles in the United States is a public health success that has provided a model for immunization programs in other parts of the developed world. Before measles vaccination was introduced in the United States in the mid-1960s, more than half a million cases of measles were re- ported each year. Once a vaccine was developed, public health officials set out to use it to control the disease, envisioning eventual global eradication. By the mid1970s, fewer than 50,000 cases were being reported annually in the United States, but a severe outbreak in Los Angeles in 1977 reminded authorities how tenuous the control of measles was. Compulsory immunization of schoolchildren followed - the first in a series of steps that led to the interruption of the transmission of measles in the United States. The most effective of these steps was probably the introduction of a second dose of measles vaccine in 1989, again in response to a 


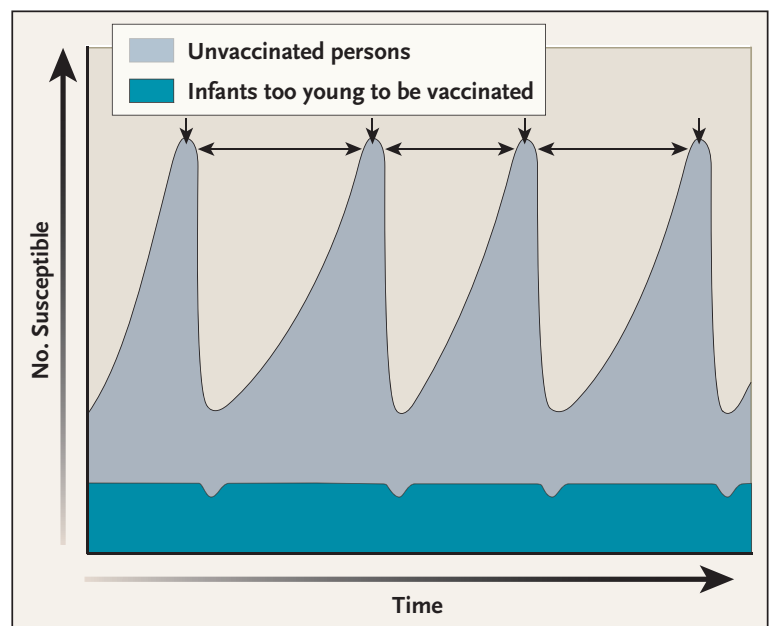

Fluctuation in the Number of Persons Susceptible to Measles in a Community Where Measles Virus Is Circulating.

In general, only a small fraction of the community will be susceptible at the time of an outbreak. The horizontal double-pointed arrows represent periods between outbreaks of measles, which vary according to levels of vaccine coverage and other factors. The downward-pointing arrows represent outbreaks of measles.

large outbreak of disease. At a meeting in March 2000, leaders in the field concluded that "measles is no longer endemic in the U.S."1

Six years later, an article in this issue of the Journal (pages 447-455) describes a 2005 measles outbreak in Indiana. Begun when a 17-yearold girl who was incubating measles returned to Indiana from Romania, the outbreak ultimately involved at least 34 persons, 1 of whom had life-threatening illness. With U.S. vaccination coverage at record levels, how could one imported case of measles lead to such an outbreak? This example illustrates the highly infectious nature of measles and the vulnerability of vaccinated communities in a world in which the virus continues to circulate.

Measles is an acute infectious disease that can affect persons of all ages but is most severe in young infants and adults. Person-to-person transmission occurs through direct contact or droplet spread, and there is some evidence that severity is related to infective dose, so that those who acquire the disease from close household contacts have the most severe disease. ${ }^{2}$ Infected persons can infect others during the prodromal period before the characteristic rash appears, creating ample opportunity for people who are incubating the disease to travel from a country where the virus is circulating, such as Romania or India (the latter being the probable origin of an outbreak in Boston in the spring and early summer of 2006), and bring the virus with them.

In any population, there are persons who are immune to measles, thanks to natural infection or immunization; persons who are susceptible to the disease; and persons with incomplete immunity who may be susceptible to mild disease. In places where measles virus circulates freely, persons with immunity appear to have their immunity boosted by intermittent exposure to the wild virus ${ }^{3}$; those who are susceptible, meanwhile, will inevitably acquire the disease unless they are immunized. After the disease has swept through a community, very few persons will remain susceptible. With time, however, new persons who are susceptible who either have not been vaccinated or did not acquire protective immunity from the vaccine they did receive - will be introduced into the community. Their numbers will gradually increase until there are enough such persons to sustain an epidemic - hence the unstable measles epidemiol- ogy found in partially vaccinated communities (see graph).

In countries such as the United States, where transmission of the wild virus has been interrupted, the rate of increase in the number of persons who are susceptible to the disease will depend on the success of the immunization program. For control to be sustained, a two-dose vaccination strategy with very high coverage is needed.

At the time of the 2005 outbreak, the level of vaccination coverage in Indiana was 98 percent for the second dose delivered to school-age children. In such a setting, it can be expected that the number of vaccinated persons who do not acquire protective immunity will be small, so the primary group of persons who are susceptible to the disease will consist of those who are unvaccinated. An important subgroup will be children who are too young to be vaccinated. These infants become susceptible when maternal immunity wears off, and they remain susceptible until they are immunized.

There is evidence that maternal immunity is declining in some countries because, without the boosting effect of circulating wild virus, mothers must rely on immunity from their own vaccinations (which were usually delivered in the second year of life). As a result, infants become susceptible to measles earlier in the first year of life, thereby increasing the number of susceptible persons present in a community at any given time. The susceptibility of young infants is of particular concern, since they are more likely than older children to have severe disease if they become infected. 
Furthermore, a generation of young adults with waning, vaccine-derived immunity may become susceptible to some extent to wild measles infection, although the role of such "secondary vaccine failures" in measles epidemiology is unclear. The longer a community goes without circulating measles virus, the more vigilant public health officials must be to maintain immunity levels in the community.

The Indiana outbreak was striking for a number of reasons. All but two of the cases occurred in unvaccinated hosts, indicating that it was the failure to vaccinate rather than vaccine failure that caused the problem. Five hundred people were reportedly present at the church gathering where the initial U.S. transmission occurred. Church officials estimated that 10 percent of them were unvaccinated, but it is unclear how they knew this or how accurate their estimate was. It seems unlikely that the person with the index case of measles could have directly infected 32 percent of all susceptible persons at the meeting, and I would suggest that the number of unvaccinated persons there may have been higher. But even 10 percent is a high proportion in a state with 98 percent coverage for the second dose of measles vaccine, despite the fluctuations in coverage that may have occurred during the lifetimes of those who became infected, most of whom were younger than 20 years old.

Community-based data such as levels of vaccine coverage represent averages for a district, state, or country; although homogeneity may seem to be implied, it never exists. Moreover, objection to immunization tends to occur in clusters, sometimes affecting whole communities. Thus, even with excellent coverage, there remain subgroups in which conditions suitable to an outbreak may persist. Large gatherings of these groups provide an ideal setting for

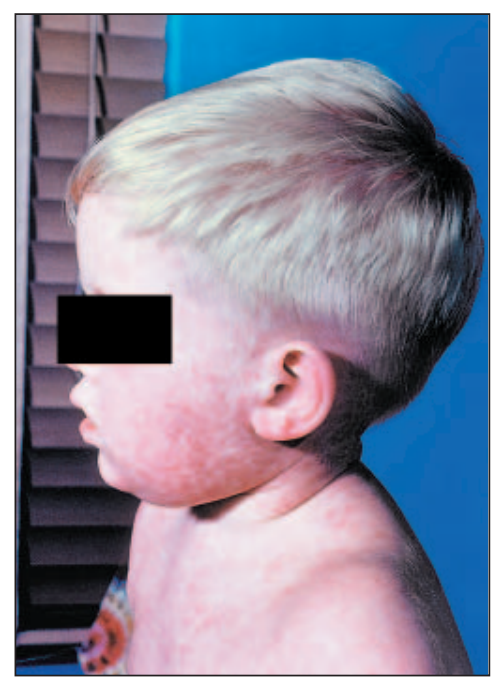

Measles Rash.

transmission, and if a person who is incubating the disease attends such a gathering, an outbreak is inevitable.

Of the two vaccinated persons who acquired measles in Indiana, one was 34 years old and had been vaccinated only once in infancy. We cannot tell whether this case represents a primary vaccine failure (a failure of the vaccine to induce immunity) or a secondary vaccine failure (the result of waning immunity). The severity of the case suggests the former, since secondary vaccine failures tend to produce mild disease. ${ }^{4}$ In addition, a 16-year-old student acquired measles despite having received two doses of measles vaccine during early childhood.

In the United States, measles vaccine is delivered in combination with mumps and rubella vaccines. Although the two-dose strategy ensures that a high proportion of vaccinees will be im- mune to measles (seroconversion after two doses of vaccine is estimated to be 98 percent), the conversion rate is lower for mumps (approximately 88 percent). Thus, the proportion of persons in a U.S. community who are susceptible to mumps is greater than the proportion who are susceptible to measles, and outbreaks, when they occur, will be larger.

More than 50,000 cases of mumps have been identified in the United Kingdom during the past few years, and more than 2500 cases have already been identified in a U.S. outbreak this year. ${ }^{5}$ About half the persons affected in the U.S. outbreak had been vaccinated, and most were young adults. Although mumps is a less severe disease than measles, it does cause substantial illness, including parotitis, orchitis, and aseptic meningitis. These outbreaks demonstrate the potential for rapid dissemination of respiratory pathogens, facilitated by air travel and crowded conditions.

Despite the vision of the public health officials of the 1960s, global eradication of measles remains a long way off. In the meantime, countries must strive to immunize as high a proportion of children as possible, using a second dose to minimize the risk of primary vaccine failure and improve coverage. Better understanding of the epidemiology of measles in vaccinated communities will permit the optimization of vaccination strategies. The importation of virus can be minimized by ensuring that travelers to and from regions where the viruses are endemic provide evidence of immunization. Yet as long as measles continues to circulate in other parts of the world, cases will continue to be imported. And as long as some groups within a given 
community respond to spurious claims about the risks of the vaccine by refusing to vaccinate their infants, further outbreaks will occur even in industrialized countries.

Dr. Mulholland is a professor in the Infectious Disease Epidemiology Unit of the Lon- don School of Hygiene and Tropical Medicine, London.

1. Katz SL, Hinman AR. Summary and conclusions: measles elimination meeting, 1617 March 2000. J Infect Dis 2004;189:Suppl 1:S43-S47.

2. Garenne M, Aaby P. Pattern of exposure and measles mortality in Senegal. J Infect Dis 1990;161:1088-94.

3. Whittle HC, Aaby P, Samb B, Jensen $H$, Bennett J, Simondon F. Effect of subclinical infection on maintaining immunity against measles in vaccinated children in West Africa. Lancet 1999;353:98-102.

4. Edmonson MB, Addiss DG, McPherson JT, Berg JL, Circo SR, Davis JP. Mild measles and secondary vaccine failure during a sustained outbreak in a highly vaccinated population. JAMA 1990;263:2467-71.

5. Update: multistate outbreak of mumps - United States, January 1-May 2, 2006. MMWR Morb Mortal Wkly Rep 2006;55:559. 63.

\section{No Refuge for the Ailing}

Ranjana Srivastava, F.R.A.C.P.

The still, oppressive heat of the afternoon seems to magnify the queue of waiting patients. A young woman separates herself from the crowd.

"Excuse me, Doctor, how long will you be?"

I answer with a flicker of annoyance, "I am not sure, but I will see you."

An hour later, it is her turn. She looks far too well, I silently judge. The well worried. She springs from her seat at the sound of her name but moves away from me toward the stairs. There, she utters a rapid command in a foreign tongue before turning apologetically toward me. Before I can greet her, a scarf-clad head comes into view. It belongs to an elderly woman physically supported by aides on each side. The aides stop on the landing, then wordlessly and cautiously lift her up to carry her into the only chair in my office. Her features are wizened, her frame more shrunken than the six decades indicated on her chart would predict. Her eyes are dull, opaque, her face a repository of apprehension, anxiety, perhaps worse. She periodically glances at her daughter, but mostly she keeps her face averted as we settle into the consultation. The daughter speaks for her.

"I am sorry if I was rude, Doctor. My mother has breast cancer, and she was waiting downstairs for a long time. We can't afford to see anyone. We need your help."

I am caught unaware.

This is a refugee clinic, run out of a makeshift health care facility as bare as any in the Third World. Volunteer physicians bring their own equipment and, often, spare drug samples. Limited numbers of doctors and meager donations mean that we can barely treat hypertension, eczema, and headache; we are rarely able to provide refugees with anything remotely resembling the standard of care.

"We don't do cancer," I want to say, keen to end the conversation right there.

"Doctor, my mother has no one. We can't pay the specialists. The emergency room bills us if it doesn't turn us away. They said you would help." Her tone combines pleading with frustration and accusation. The mother winces with pain. The daughter solicitously measures out some morphine. As the patient swallows it with a wry face, the daughter murmurs, "This is the last morphine. We have to wait now until ...."

"Until . . . ?"

"Until my husband's disability pension arrives."

She brusquely runs her hand across the involuntary tears that have started. Her mother's hand surreptitiously reaches out to comfort her. In that moment, the enormity and heartache of the situation descend on me, and Mrs. Habib becomes my patient.

Having lost all the male members of her family through war, Mrs. Habib arrived from Afghanistan to live with her only daughter, an Australian resident. Here, her application for refugee status was denied on grounds of insufficient evidence, thus denying her access to any form of government support, including food, shelter, employment, and health care. While the decision is appealed on humanitarian grounds, a process that can take years, the restrictions continue. Reliance on the family's small earnings worked until she developed cancer. A public hospital provided her with a free mastectomy but no follow-up. Now she is riddled with painful bony metastases. I read letters 